\title{
Filtering and Processing IR Images of PV Modules
}

\author{
S. Vergura, O. Falcone \\ Dipartimento di Elettrotecnica ed Elettronica \\ Politecnico di Bari \\ Via E. Orabona 4, 70100 Bari (Italy) \\ Phone/Fax number:+39 080 5963590, e-mail: vergura@poliba.it
}

\begin{abstract}
The aim of the paper is to propose a procedure to process InfraRed (IR) images of PV modules. In fact, when an IR image is acquired by thermo-camera, three possibilities can happen. The first one regards the acquisition of an IR image of a well-working PV module; in this case the IR image reveal no anomaly. The second case regards the acquisition of an IR image of a bad-working PV module; then, the IR image reveal clearly the anomaly. The third one regards the acquisition of an IR image, which gives no clear information about the health of the PV module. In this last case, the uncertainty can be totally or partially removed only after filtering and processing the IR image. Proposed procedure and tools have been applied on IR images of real PV modules.
\end{abstract}

\section{Keywords}

Infrared analysis, median filter, gaussian filter, edge detection

\section{Introduction}

When a PV plant is built up, it is very important to monitor its energy performance, because of the very low efficiency. In fact the efficiency range goes from $6 \%$ to $17 \%$ for commercial PhotoVoltaic (PV) modules, depending on the used material. Then, an efficiency decrease of some per cent affect strongly its energy performance. The energy decrease of a PV plant can depend on the PV modules (one or more) or on the Balance Of System (BOS). When the anomaly or failure depends on a PV module, two case can happen: the PV module produces no energy or PV module produces less energy than the expected one. In the last case, an effect of the reduced energy production is an abnormal heating of the PV module. Usually the abnormal heating of an area of the solar device is due to the presence of defects, called shunts [1]. Defects act as a parasitical resistance connected to the cell; they may be due to some fabrication steps or else to the features of the substrate itself or to the normal/abnormal ageing. The defected area shows a temperature increase (hot spot) with respect to the remaining cell that could be small or strong depending on different factors. Often, it may be very difficult to highlight hot spots related to defects because of their reduced dimensions. The advanced thermography allows performing an efficient, systematic and non destructive [2] investigation on typical defects in solar cells [3]-[4] and the identification of possible hot spots that may also be conducted in an automated way [5]. Some typical defects have been implemented and simulated in COMSOL environment [6]. Nevertheless, sometimes it is not possible to obtain sufficient information from an IR image because thermo-cameras have low resolution. In fact, an high resolution thermocamera has a resolution of only $640 \times 480$ pixel. Moreover, sometimes IR images are dirty, then it is not easy to understand the cause of an overheating. For these issues a proper processing of the acquired IR images has to be performed [7]. The paper proposes tools to preprocessing and processing IR images, when the acquired image cannot give satisfactory information about the health of a PV module. Particularly, the paper proposes the application of both median and Gaussian filters and then the edge detection. The procedure has been implemented in a Matlab Graphical User Interface (GUI) in order to fast the whole procedure.

\section{Proposed algorithm}

The main functions of the proposed algorithm are reported in Fig. 1. Let us study the single steps.

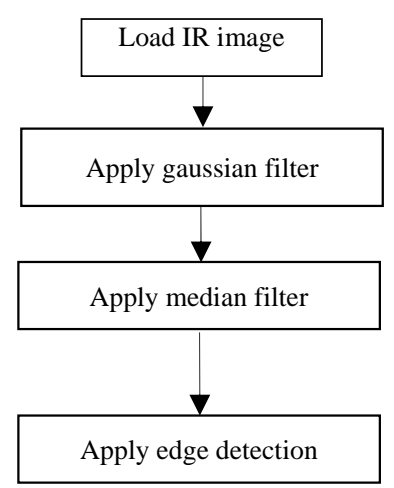

FIG. 1. THE PROPOSED ALGORITHM 


\section{A. Load IR image}

The IR images are acquired and stored in an internal memory of the thermo-camera or in memory card or in PC, if directly connected to the thermo-camera. Usually, each acquisition consists of a file containing not only the IR images but also the information about the settings of the thermo-camera (emissivity, relative humidity, distance from the target and so on); and usually this information is managed by a specific software of the thermo-camera, protected by copyright. Then, sometimes it is difficult to take only the IR image from the proprietary file in order to process it. When it is possible, the image has a standard format .jpg or .tiff or other. In some cases the acquisition file can be saved directly in Matlab format .mat, as it happens for Flir Systems. Anyway, standard format of images can be easily imported in Matlab environment.

At this point, the IR image can be loaded in Matlab environment by a simple routine. Each IR image is stored in a 3D structure, in which $\mathrm{X}$ and $\mathrm{Y}$ coordinates allow locating the position of each pixel, while $\mathrm{Z}$ coordinate represents the intensity value.

Load Thermogram routine can be run from the block n. 2 of the implemented GUI (Fig. 2). As it can be seen, it is also possible to Reload a 3D image, if it has been previously utilized.

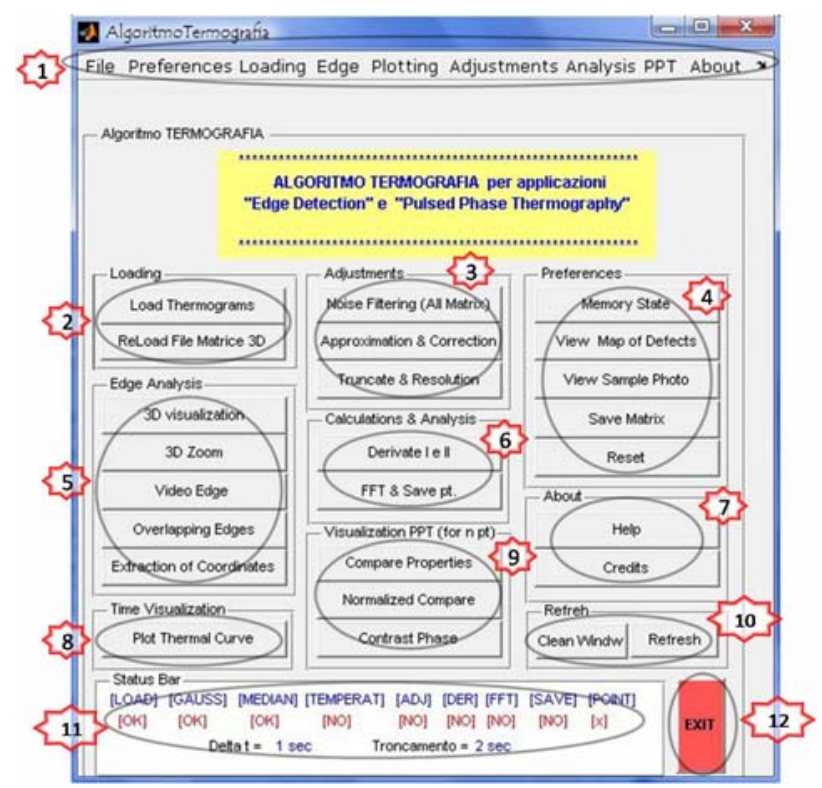

FIG. 2. GUI OF THE PROPOSED ALGORITHM.

\section{B. Gaussian filter}

This routine can be applied by using Noise Filtering of the block n. 3. It consists in applying a $2 \mathrm{D}$ gaussian function to the IR image:

$$
G(x, y)=G(x) G(y)=\frac{1}{2 \pi \sigma^{2}} e^{-\frac{\left(x^{2}+y^{2}\right)}{2 \sigma^{2}}}
$$

The resulting image is the convolution between the original IR image and the gaussian filter. Good value of the standard deviation $\sigma$ for an IR image of PV module is
3, as it will be shown successively. At this point the modified IR image can be saved (Save Matrix in block n. 4) to avoid the same operations for the successive processing.

\section{Median filter}

This routine can be applied by using Noise Filtering of the block n. 3. It consists in substituting the value of each pixel by the median value of the closer ones. Median value is the value that satisfies the following condition:

$$
\sum_{i=1}^{N}\left|x_{\text {med }}-x_{i}\right| \leq \sum_{i=1}^{N}\left|x_{j}-x_{i}\right|
$$

for $j=1 \ldots N$.

The resulting image is the convolution between the original IR image and the median filter. Good value of the kernel for an IR image of PV module is 3 or 5 , as it will be shown successively. At this point the modified IR image can be saved (Save Matrix in block n. 4) to avoid the same operations.

\section{Edge detection}

This routine can be applied by using Edge Analysis (block n. 5). It consists in applying a filter based on the derivative of a gaussian function. When the image is strongly filtered, a bad edge detection is obtained. This is the reason to choose $\sigma=3$ and $\boldsymbol{k}=3 \div 5$ for the previous gaussian and median filters. This last step allows to apply Canny method for the edge detection and to extract (in Matlab window) the coordinates of the edges; moreover, the routine gives the edge detection superimposed on the filtered IR image.

GUI contains also a status bar (block n. 11) that gives information about the progress of the whole procedure. Other several functions (implemented in the proposed GUI) can be applied to IR images to extract other information, but they regard a time series acquisition of IR images. These typologies of acquisition are not treated in this paper.

\section{Results}

Several acquisitions have been made on $20 \mathrm{kWp} \mathrm{PV}$ plant, located in Bari, Italy. Following settings have been imposed on the thermo-camera Flir A40-M, with resolution of 320x240 pixel:

- Emissivity = 0.85, typical for the glass of PV plants;

- Environment average temperature $=10^{\circ} \mathrm{C}$;

- Relative average humidity $=54 \%$; with this value the effect of the air humidity on the transmissivity can be offset.

- Distance $=2 \mathrm{~m}$; this is the distance between the front lens of the thermo-camera and the surface of the object under investigation. This value has to be set up to rectify the radiation absorption between the camera and the target and the transmissivity reduction due to the distance. 
Three cases are proposed in this paper. Each case report an IR image of a defected PV module, 3D plots and several filtered images with different values of the filter parameters (sub-sections A, B, C, respectively). An edge detection by Canny method is reported only for the third case (sub-section D).

\section{A. $1^{\text {st }}$ case}

Figure 3 reports an IR image of a real PV module. The black points are the anchor clamps of the PV module to the iron structure. Figure 4 reports a 2D plot of the Fig. 3, where the $x$ and the $y$ axes represent the dimensions of the analyzed IR image (320x240 pixel). Figure 5 reports the 3D plot of Fig. 3, where the $z$ axis show the temperature values. Note as in the upper left side of the module there are two cold cells that surely are in a cut off situation (they do not produce energy), thus resulting into a shunt.

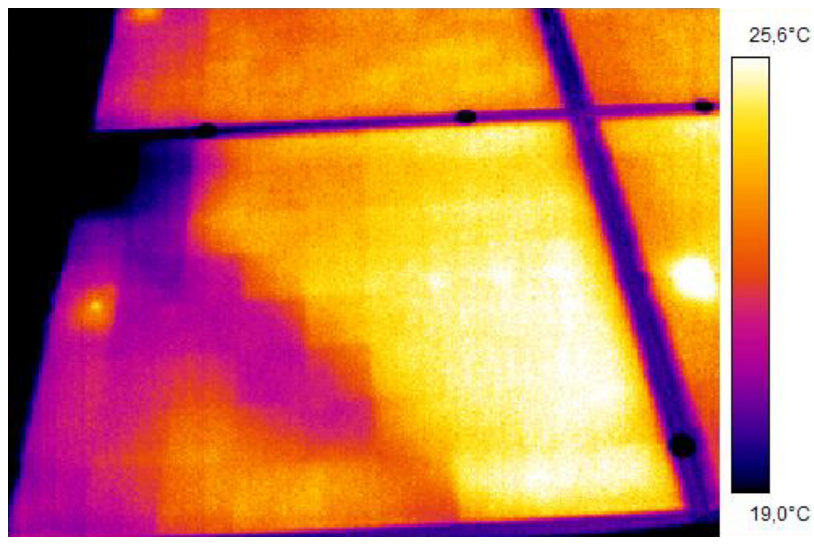

FIG. 3.IR IMAGE OF THE FIRST CASE.

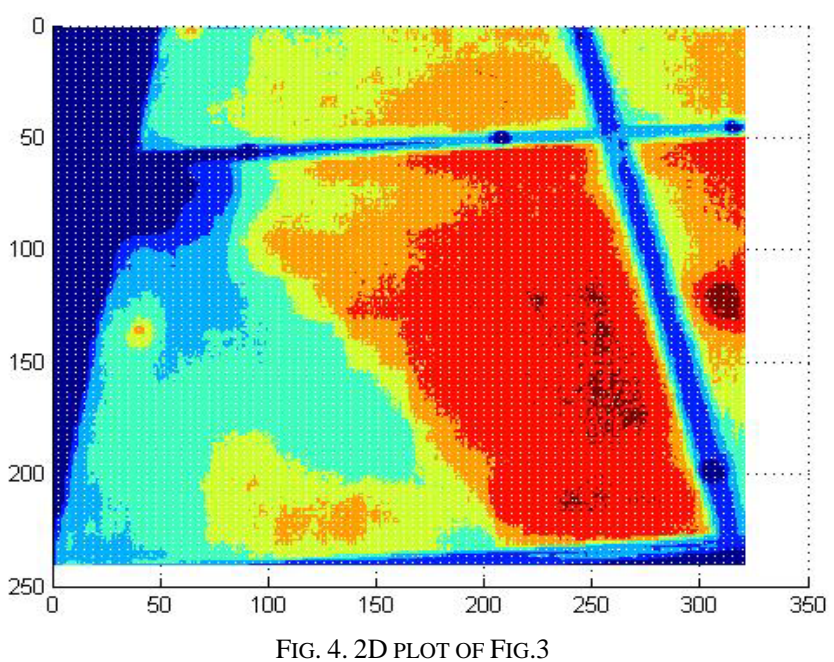

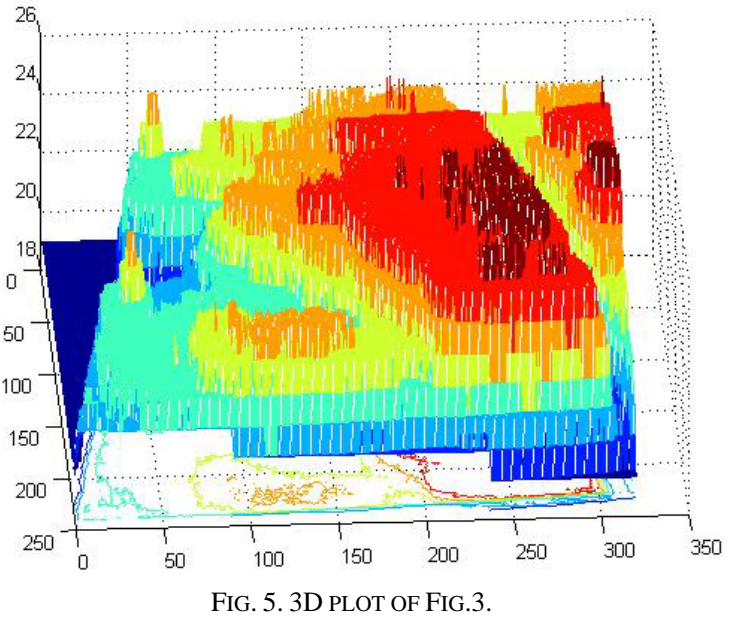

After IR images have been loaded they have to be filtered. Different values for the standard deviation $\sigma$ for the gaussian filter and the kernel $k$ for the median filter have been set, depending on the PV module under study. The following Figures 6 and 7 show the 3D plots of Fig. 3 with two different values for $\sigma$ and $\mathrm{k}$.

Focusing attention on the circled areas of Fig. 7 it can be noted that information about hottest areas of the module has been lost with respect to Fig. 6, where hot areas are still visible. This evidence shows as it is not useful to increase the value of the filter parameters because this makes flats the curves available in the 3D thermo-gram. In fact filters are designed to eliminate noises on the image, this resulting in leveling the temperature profile.

Figures 8 and 9 report a 3D and a 2D plot with the same values of $\sigma$ and $k$, respectively. It is possible to see as considering the values $\sigma=5$ for gaussian filter and $k=$ 5 for the median one, the edges of areas with different temperatures may be lost.

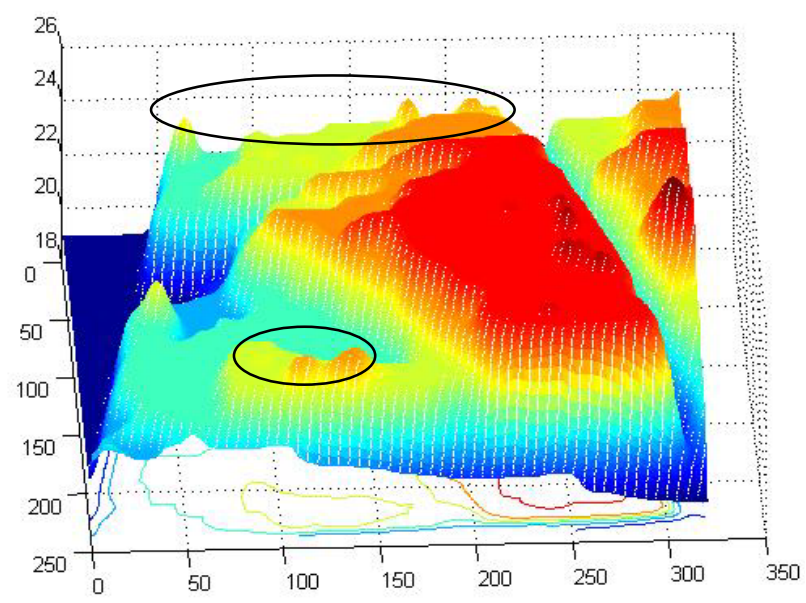

Fig. 6. Filtered 3D PLOT OF Fig.3, $\sigma=3$ AND $k=3$. 


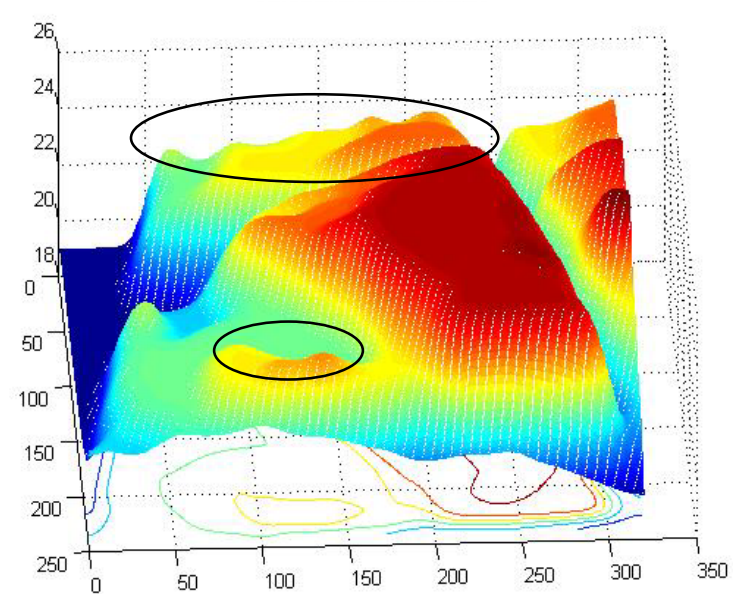

Fig. 7. Filtered 3D PLOT OF Fig.3, $\sigma=3$ AND $k=5$.

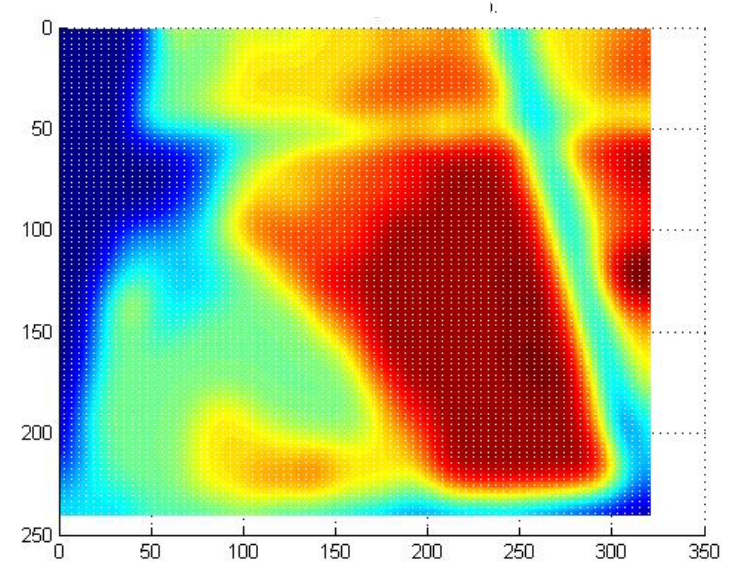

FIG. 8. 2D PLOT OF FIG.3, WITH $\sigma=5$ AND K $=5$.

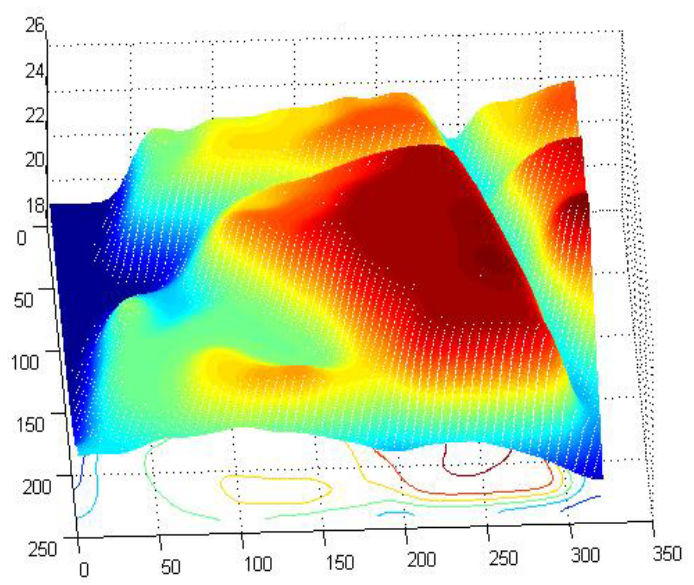

FIG. 9. 3D PLOT OF FIG.3, WITH $\sigma=5$ AND K $=5$.

The edge loss is well visible in the $3 \mathrm{D}$ plot as well as in the $2 \mathrm{D}$ plot. At this point it is important to clarify that the proposed algorithm allows developing the $2 \mathrm{D}$ plot for each case, but 3D plots are more accurate than 2D ones to localize hot spots; then, in the following only 3D plots will be reported.

\section{B. $2^{\text {nt }}$ case}

Figures 10 reports the IR image for the second case under investigation.

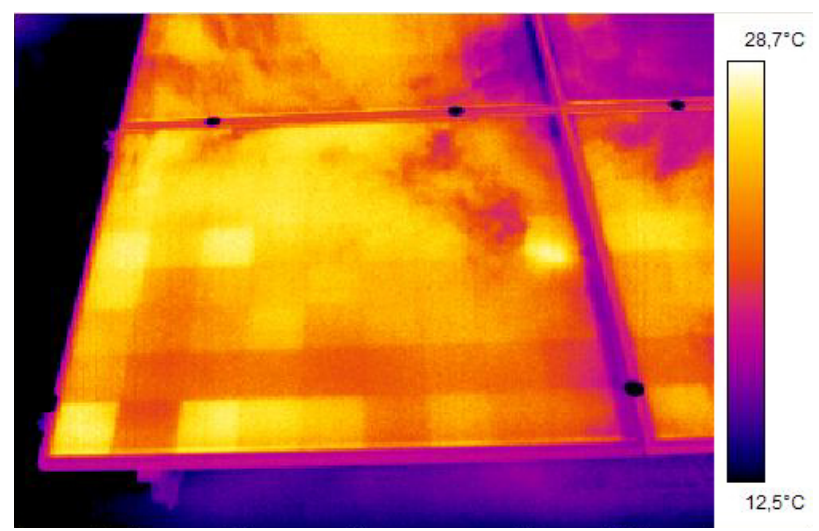

FIG. 10. IR IMAGE OF THE SECOND CASE.

The Figure 11 reports the 3D plot of Figure 10 while Figures 12 and 13 show the filtered 3D plots. $\sigma$ has been set up equal to 3 in both cases while $k$ have been set up equal to 3 in the first case and to 5 in the second one.

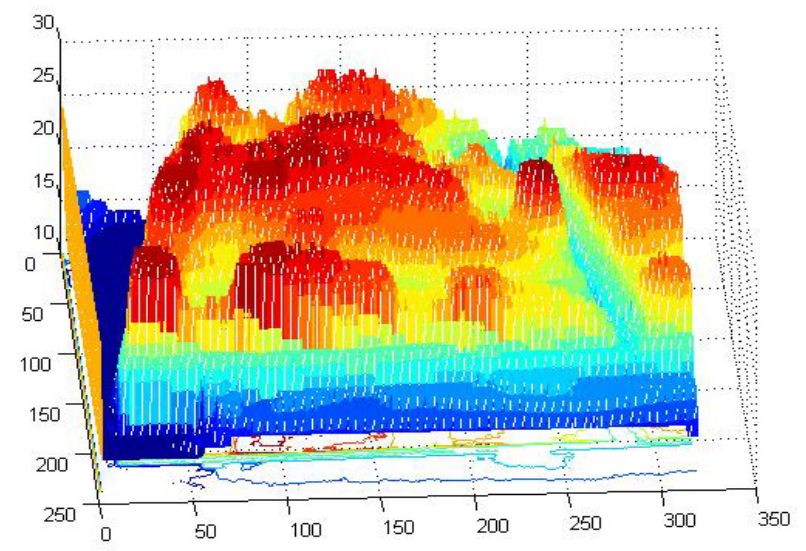

FIG. 11. 3D PLOT OF FIG. 10.

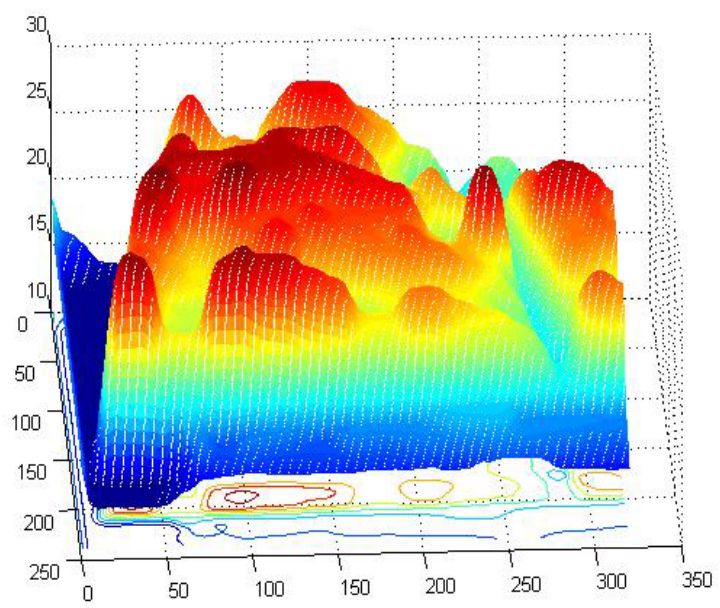

FIG. 12. FILTERED 3D PLOT OF FIG.10, WITH $\sigma=3$ AND K $=3$. 


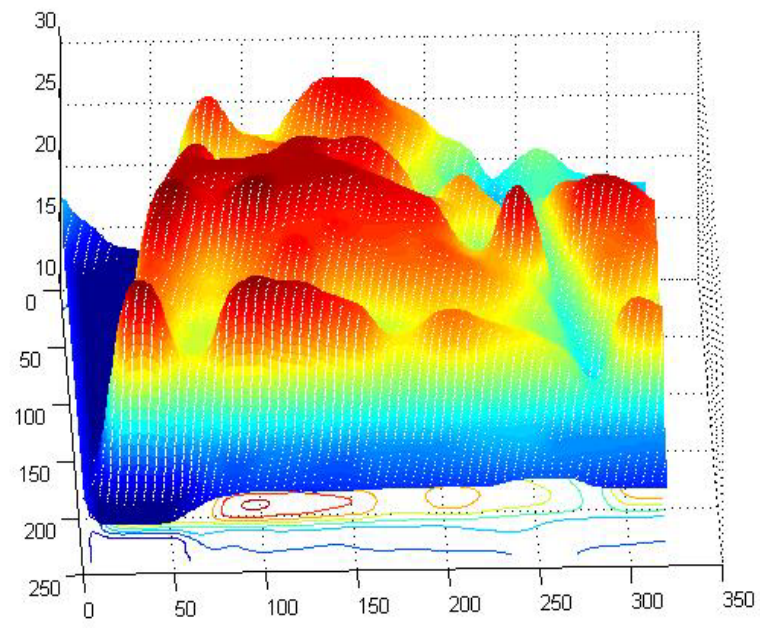

FIG. 13. Filtered 3D PLOT OF FIG.10, WITH $\sigma=3$ AND K $=5$.

Looking at the thermo-gram it is possible to highlight as some cells show a temperature difference with the respect to the remaining cell of about $17^{\circ} \mathrm{C}$. As reported in [6], this temperature increase can cause a reduction of about $10 \%$ of the produced energy.

$$
\text { C. } 3^{\text {rd }} \text { case }
$$

Fig. 14 reports the IR image of a defected PV module, where the hot spot on the right hand-side represents the overheating due to the junction box, located on the back of the module. Moreover, it can be noted that 18 adjacent cells and the cell in the lower left corner are overheated and then they produce less energy than the expected one. Fig. 15 reports a $3 \mathrm{D}$ plot of the IR image, but rotated of about $90^{\circ}$.

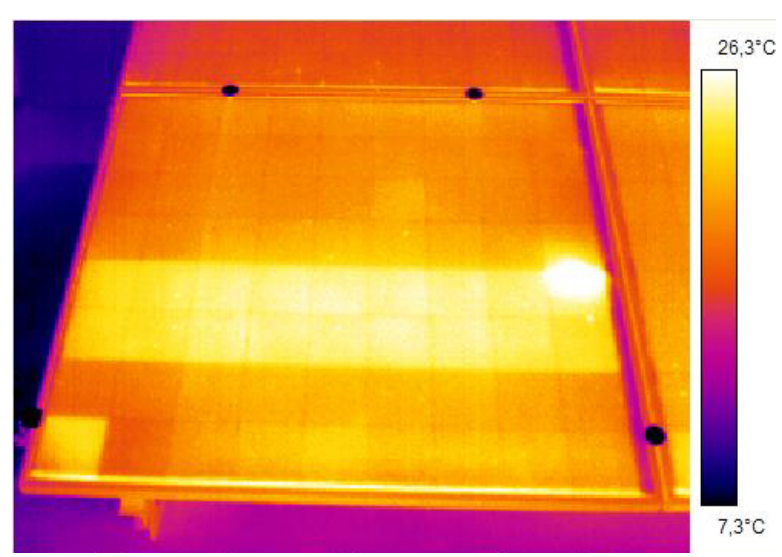

FIG. 14. IR IMAGE OF THE THIRD CASE.

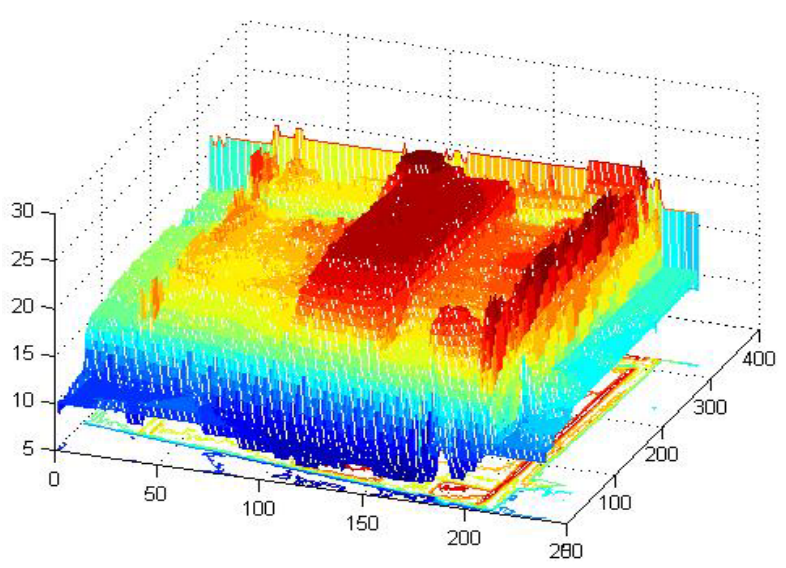

FIG. 15. 3D PLOT OF FIG.14, BUT ROTATED OF ABOUT $90^{\circ}$.

Fig. 16 is the filtered 3D plot of Fig. 14, in which $\sigma=3$ (for gaussian filter) and $k=3$ (for median one) have been imposed. Fig. 17 is the filtered 3D plot of Fig. 14 , in which $\sigma=3$ (for Gaussian filter) and $k=5$ (for median one) have been imposed. Even if they seems almost equal, different results are obtained by the successive edge detection. Other values of $\sigma$ and $k$ give bad results after edge detection is applied.

It is possible to highlight as Fig. 16 allows detecting some peaks not revealed in Fig. 17; nevertheless, the 18 hottest cells and the connection box hot spot are visible in both images.

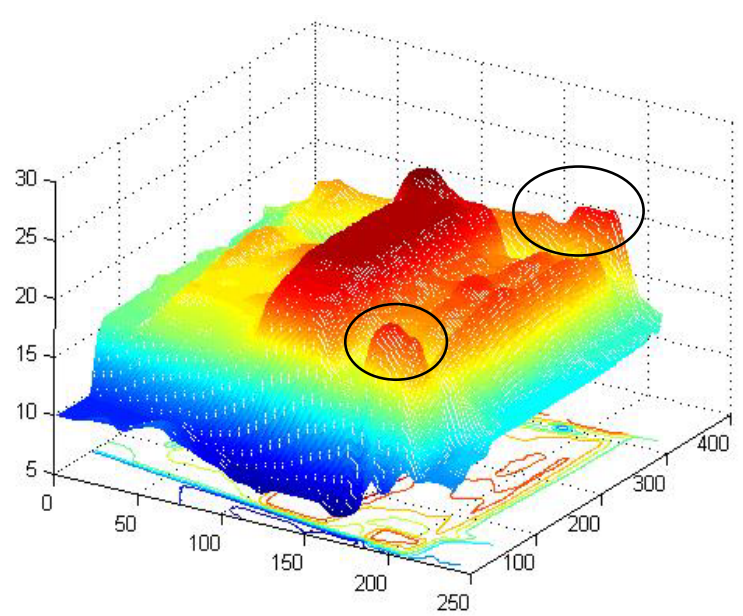

FIG. 16. Filtered 3D PLOT OF Fig.14, $\sigma=3$ AND $k=3$.

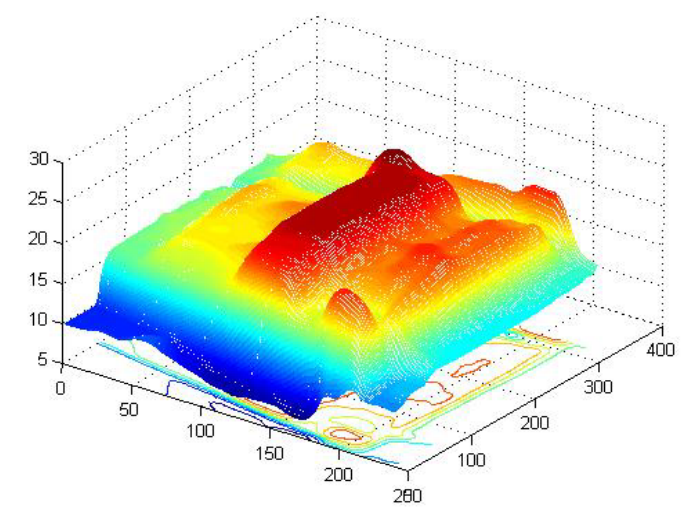

Fig. 17. Filtered 3D PlOT OF Fig.14, $\sigma=3$ AND $k=5$. 


\section{Edge detection for the $3^{\text {rd }}$ case}

After IR images have been processed it is possible to highlight the areas of the thermo-gram characterized by a higher temperature, in which a defect may probably be located. The edges of the IR image can be extracted by means of Canny method. The proposed algorithm allows developing a superposition of the extracted edges on a gray scale image of the IR itself. Two parameters are to be set up in this last step: the noise threshold, whose maximum value can be 10 , and the identification threshold whose maximum value can be 1 .

If the Canny method is put on a not filtered IR image, the edge detection allows selecting a lot of edges, some of them unnecessary. Otherwise, if it is put on a filtered image, fewer edges are present; this allows identifying the most significant areas.

Fig. 18 represents, in gray scale, the filtered image of Fig. 14 ( $\sigma=3$ and $k=5$ ) with the superimposed Canny edge.

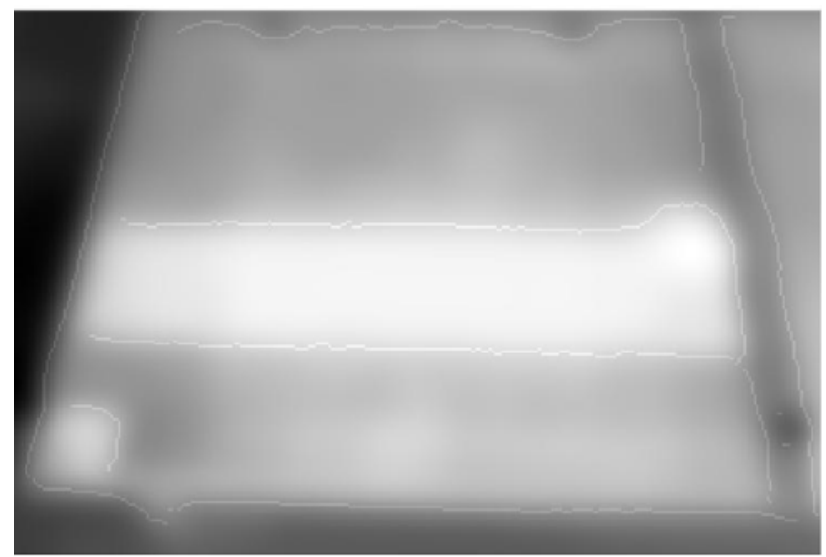

FIG. 18. FILTERED IR IMAGE OF FIG. 14 AND CANNY EDGE.

The identified edges circle the hot areas of the PV module: the cell on the lower left side and the 18 hottest adjacent cells. In fact these areas are the most significant elements of the investigated module.

Finally, Figures 19 reports the same gray scale IR image of Fig. 14, in which the increase of the contrast leads to a more blurred image. Nevertheless, this aspect does not affect a correct detection of the edges by means of Canny method applied to a filtered image, as Fig. 20 shows.

\section{Conclusions}

The paper has proposed a procedure to process IR images of PV modules. Simulation results have shown that the best results are obtained setting the values for $\sigma$ and $\mathrm{k}$ equal to three. Increasing the value of standard deviation and kernel, temperature differences are less visible and information can be lost.

Effective values for $\sigma$ and $k$ have been presented in order to obtain clear IR images before the application of the edge detection. Canny method has given good results for IR images of PV modules. In the opinion of the authors, other information about the correct/incorrect operation of PV modules can be obtained by observing the thermal behavior of the PV modules during the first minutes after the switching on or the switching off.

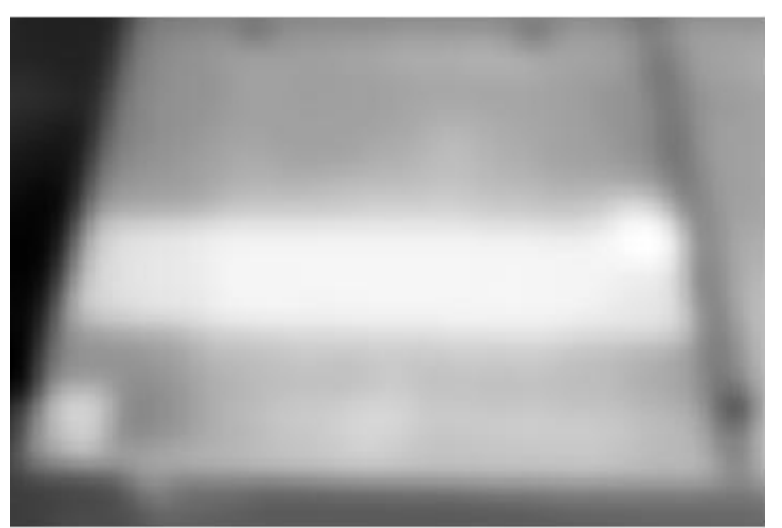

FIG. 19. FILTERED IR IMAGE OF FIG.14 WITH INCREASED CONTRAST.

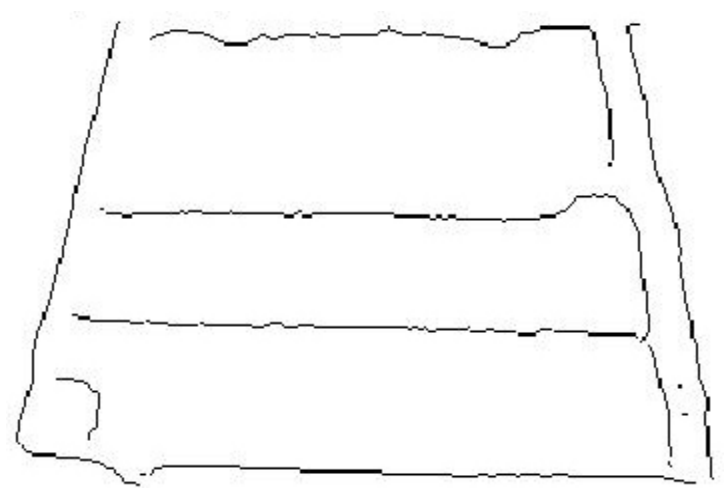

Fig. 20. NEGATIVE CANNY AFTER FILTERING $(\sigma=3, \mathrm{~K}=5)$.

\section{REFERENCES}

[1] M. A. Quintana, D. L. King, T.J. McMahon, C. R. O. Sterwald, Commonly observed degradation in field-aged photovoltaic modules, Proceedings of 29th IEEE photovoltaic specialists conference, 2002, pp. 1436-1439.

[2] Maldague X.P., "Theory and Practice of Infrared Technology for Nondestructive Testing”, John Wiley \& Sons, N. Y, 2001.

[3] Breitenstein O., Langenkamp M, Rakotoniaina JP, Zettner J., “The imaging of shunts in solar cells by infrared lock-in thermography". Proceedings of the 17th European Photovoltaic Solar Energy Conference, Munich, 2002; pp. 1499-1502.

[4] Breitenstein O., Rakotoniaina JP, Al Rifai M.H., 2003, "Quantitative evaluation of shunts in solar cells by lock-in thermography", Progress in photovoltaics research and application, 11, 515-526.

[5] Ying-Chieh Chou, Leehter Yao, "Automatic Diagnostic System of Electrical Equipment Using Infrared Thermography", International Conference of Soft Computing and Pattern Recognition, December $4^{\text {th }}-7^{\text {th }}$, Malacca, Malaysia , 2009

[6] G. Acciani, O. Falcone, S. Vergura, "Typical defects of PV-cells", IEEE-ISIE 2010, Bari, Italy, $4^{\text {th }}-7^{\text {th }}$ July 2010, pp. 2745-2749.

[7] Chen Liu, X. Maldague, "Relationship of Object Surface Geometry on Fourier-Transformed Phase and Amplitude Images in Infrared Thermography”, University of Laval Québec, 2006. 